\title{
Determinación del momento de corte de la flor en limonio (Limonium sp.) a partir de seis estados fenológicos
}

\section{Determination of timing of flower cut in sea lavender (Limonium sp.) starting from six phenological stages}
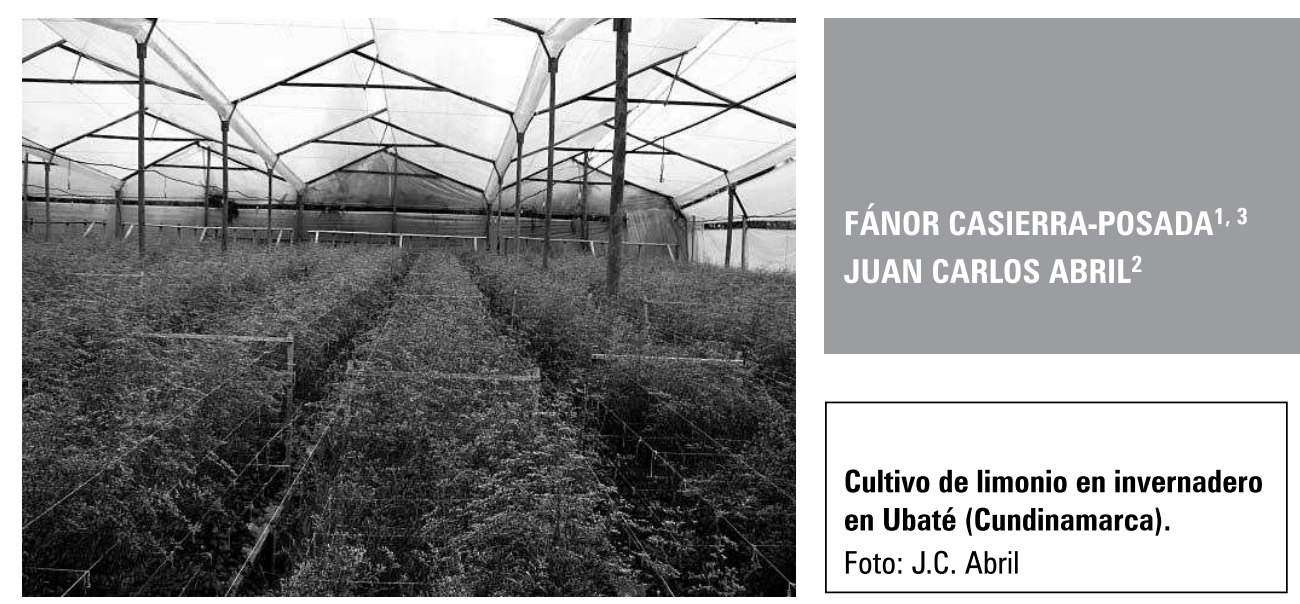

\section{RESUMEN}

La fenología es el estudio de los tiempos de los fenómenos naturales recurrentes en organismos vivos. Concierne principalmente a las épocas de ocurrencia, por primera vez, de los eventos naturales en el ciclo de las plantas. Las observaciones fenológicas las usan los agricultores desde hace siglos para maximizar la producción agrícola, mediante la predicción de la época estacional de la cosecha. Es muy poca la disponibilidad de información relacionada con la planificación de la cosecha de la flor usando los estados fenológicos como referencia, en la producción de flores de corte. Se realizó un trabajo en invernadero en Ubaté (Cundinamarca, Colombia), para una mejor comprensión del crecimiento de los tallos a partir de seis estadios fenológicos diferentes hasta el momento de corte en cinco variedades de limonio 'Supreme white light', 'Supreme blue night', 'Safora dark blue', 'Safora blue' y 'Beltlaard'. Se identificaron seis estadios fenológicos, y a partir de ellos se midió semanalmente la altura del tallo floral. La altura final de los tallos fue de 132,3; 130,1; 102,1; 85,6 y $113,2 \mathrm{~cm}$ para 'Supreme white light', 'Supreme blue night', 'Safora dark blue', 'Safora blue' y 'Beltlaard', respectivamente. Con la información colectada se calcularon las ecuaciones de regresión para el crecimiento. Para todas las variedades, a partir de cada estadio fenológico, se encontró un modelo cúbico con alto coeficiente de determinación $\left(R^{2}=0,99\right.$ a 1,0$)$. Las ecuaciones se pueden usar para la predicción del momento de corte para las variedades evaluadas, con propósitos prácticos.

1 Grupo de Investigación Ecofisiología Vegetal, Facultad de Ciencias Agropecuarias, Universidad Pedagógica y Tecnológica de Colombia, Tunja (Colombia).

2 Facultad de Ciencias Agropecuarias, Universidad Pedagógica y Tecnológica de Colombia, Tunja (Colombia).

3 Autor para correspondencia. fanor.casierra@uptc.edu.co 


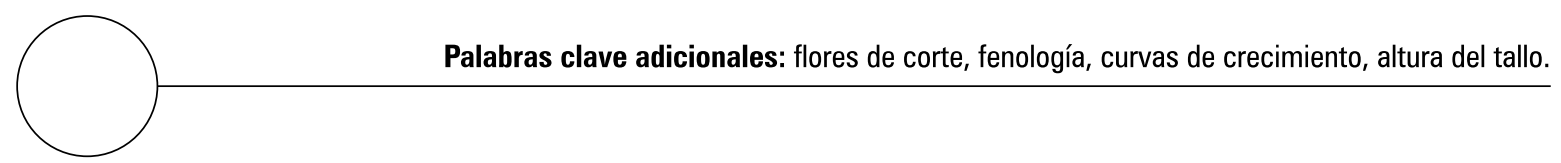

Abstract

Phenology is the study of timing of natural phenomena recurring in living organisms. It has been principally concerned with the dates of first occurrence of natural events in the plant life cycle. Phenological observations have been used for centuries by farmers to maximize crop production by predicting the seasonal harvest time. Very little information is available on the planning of harvest of cut flowers using phenological stages as reference in cut flower production. In order to understand better the process of stem growth of five sea lavender varieties starting from six different phenological stages up to the cutting time, a study was carried out in greenhouse in Ubaté (Cundinamarca, Colombia). Plant material were sea lavender varieties 'Supreme white light', 'Supreme blue night', 'Safora dark blue', 'Safora blue' and 'Beltlaard'. Six phenological stages were identified and starting from these stages, the growth of plant flower stem was weekly measured. The final stem height were 132.3, 130.1, 102.1, 85.6 and $113.2 \mathrm{~cm}$ for 'Supreme white light', 'Supreme blue night', 'Safora dark blue', 'Safora blue', and 'Beltlaard', respectively. With information collected, the regression equations for growth were calculated. For all varieties from each phenological stage a cubic model with high coefficients of determination $\left(R^{2}=0.99\right.$ to 1.0$)$ was found. The equations could be used for predicting the cut time for evaluated varieties for practical purposes.

Additional key words: cut flowers, phenology, growth curves, stem height.

Fecha de recepción: 08-07-2008

Aprobado para publicación: 02-12-2008

INTRODUCCIÓN

Limonium es un tipo de planta que produce una flor de corte utilizada en la elaboración de bouquets, en los que se combinan especies de colores variados, lo que otorga al ramo un valor agregado, razón por la cual, las compañías productoras de flores cultivan diferentes especies de este género con miras a lograr un mayor contraste en el colorido del bouquet (Chamorro et al., 2007). El área destinada en Colombia, al cultivo de las flores, alcanzó 7.266 ha en 2007, en donde $79 \%$ de la producción tuvo lugar en la Sabana de Bogotá, $17 \%$ en Antoquia y en la región central y occidental del país se cultivó el 4\% del área. De los productos cultivados, los bouquets están representados con un $32,48 \%$ de la producción nacional de flores de corte (Asocolflores, 2007).
En especies de Limonium se han desarrollado investigaciones orientadas a determinar la respuesta de las plantas a la temperatura y al fotoperiodo, encontrándose en $L$. sinuatum (statice) que esta especie no se ve afectada por el fotoperiodo, pero sí por la temperatura (Shillo, 1976; Escher, 1996). De igual manera, se ha encontrado en plantas de limonio (L. gmelinii) que la exposición de las plantas a bajas temperaturas en un estado adecuado del crecimiento, son necesarias para la floración; posteriormente, condiciones de día largo incrementan la tasa de floración, luego de que las plantas han evacuado su requerimiento de baja temperatura (Farina et al., 2000). En otros temas relacionados, se estudiaron el impacto de la cosecha de flores de Limonium carolinianum 
sobre las posibilidades de supervivencia de las plantas (Baltzer et al., 2002) y Doi y Reid (1995) mejoraron la vida poscosecha en híbridos interespecíficos de Limonium

Los trabajos mencionados, desarrollados en especies de Limonium tienen gran relevancia, sin embargo, se hace prioritaria la generación de información que contribuya al desarrollo de la floricultura nacional, y en especial, de las plantas de limonio cultivadas bajo las condiciones colombianas, dado que los trabajos realizados en esta área son bastante escasos. Al respecto, CasierraPosada y Moreno (2007) realizaron un estudio en el que evaluaron el comportamiento de plantas de limonio expuestas a diferentes niveles de sombrío. Los autores hacen referencia a la alta sensibilidad de las plantas a la reducción de la luz.

A pesar de que el crecimiento y desarrollo de las plantas es un proceso continuo en el tiempo, resulta posible discernir en él, una serie de etapas, que si bien, pueden tener diferencias aún en individuos de la misma especie, posee una regularidad que permite sacar conclusiones, cuando estas fases se registran por periodos prolongados de tiempo. El registro fenológico de una especie durante un tiempo más o menos considerable permite conocer las fechas probables en que se producirán las fases de crecimiento y desarrollo, así como también, las fechas límites (tempranas o tardías) y la frecuencia de las mismas, lo que posibilita el cálculo de la probabilidad porcentual de ocurrencias y la elaboración de mapas isócronos de la ocurrencia de las diferentes fases, lo que resulta de gran interés práctico en biología, agricultura y agrometeorología (Fuentes, 1992a, 1992b).

Por otro lado, para efectuar la comparación entre tipos de plantas o para realizar estudios de predicción, se debe elegir un estado de desarrollo o fenológico, y a partir de él, se deben realizar las mediciones requeridas para el logro del objetivo propuesto. Por lo regular, no se parte de una fecha calendario fija, puesto que se podría incurrir en errores, dado que a pesar de que la sucesión de los estados fenológicos es segura, puede variar en la duración de los intervalos en relación con el ambiente en el que se desarrollan las plantas (Sitte et al., 2004). Por tanto, cuando se aplica la fenología en plantas, se registra la dinámica del desarrollo, haciendo especial hincapié en la sucesión de los diferentes estadios, más que en la influencia de los factores que los producen (Ghersa y Holt, 1995).

En cuanto a la aplicación de los estudios fenológicos a la producción de flores de corte, se puede tomar en consideración los realizados en Anthurium andreanum en donde se determinaron los componentes del rendimiento para la producción de la flor. Las plantas presentan un crecimiento monopodial en la fase vegetativa, seguido de una fase simpodial, con producción de una flor por hoja (Dufour y Guérin, 2002). Con mayor exactitud que en el caso de anturio, los estadios fenológicos se han determinado y se han usado en cultivos de rosas y claveles de corte. Además de los estadios fenológicos, se determinaron algunos índices de crecimiento como la tasa de crecimiento del cultivo, el índice de área foliar, la tasa relativa de crecimiento y la tasa de asimilación neta, los cuales son una herramienta importante para la toma de decisiones en este tipo de cultivos (Chaparro et al., 2006; Cárdenas et al., 2006; Farías et al., 2006). A pesar de la importancia de la aplicación de estudios de fenología correlacionados con el rendimiento, orientados a la predicción y ajuste de épocas de corte de la flor para cumplir con las temporadas de máxima demanda por parte del mercado internacional, son muy pocos los reportes al respecto en floricultura.

El propósito del presente estudio fue la determinación del tiempo en que se realizaría la cosecha en cinco cultivares de limonio (Limonium sp.), cuando se parte de seis estados fenológicos diferentes, bajo condiciones de invernadero. 


\section{MATERIALES Y MÉTODOS}

La investigación se realizó en un cultivo establecido en la empresa Flores Ubaté Ltda., ubicada a 5०18'24" N, 7348'52" W y 2.560 msnm. Las plantas tenían cerca de 19 semanas de plantadas y fueron manejadas bajo condiciones de hidroponía con cascarilla de arroz tostada (80\% de quemado en horno). Los fertilizantes para la presiembra se tomaron a partir de fuentes simples y se aplicó por cama ( $30 \times 1 \mathrm{~m})$ la siguiente mezcla: sulfato de calcio $3,5 \mathrm{~kg}$; carbonato de calcio 2,5 $\mathrm{kg}$; óxido de magnesio $0,9 \mathrm{~kg}$; sulfato diamónico $1 \mathrm{~kg}$ y Rafos (Abocol®) 1,5 kg. La mezcla para la solución aplicada por el riego estuvo compuesta por (mg L-1): N 145; Ca 100; P 20; K 120; Mg 30; Mn 0,5; Fe 2,5; Cu 0,5; Zn 0,5 y B 1,5. La solución se suministraba en dos pulsos por día y cada pulso estaba compuesto por $40 \mathrm{~L}$ por cama. Durante el ensayo se registró en promedio, una temperatura de $16,5^{\circ} \mathrm{C}$, con mínima de $8^{\circ} \mathrm{C}$ y máxima de $25^{\circ} \mathrm{C}$. El material vegetal correspondió a variedades de Limonium sp., utilizadas comúnmente en la producción de bouquets, como son: 'Supreme white light', 'Supreme blue night', 'Safora dark blue', 'Safora blue' y 'Beltlaard'. Las cuatro primeras variedades fueron suministradas por la compañía Danziger- "Dan" Flower Farm, la última por Ball SB L.C.

Se tomó una cama por variedad y de esta, seis brotes en cada uno de los estadios fenológicos seleccionados. Cada estadio fenológico tuvo cinco réplicas dentro de la misma cama y se les llevó un seguimiento de crecimiento cada semana desde el punto de partida de cada brote hasta el momento que fue cortado para su respectivo proceso de poscosecha.

A los diferentes estados fenológicos de brotes se les asignó un nombre, de la siguiente manera: estado 01 ó punto mini, estado 02 ó punto pequeño, estado 03 ó punto medio, estado 04 ó punto espiga, estado 05 ó rayando color, y estado 06 ó punto marrón (figura 1). El estado 01 ó punto mini correspondió a la morfología del

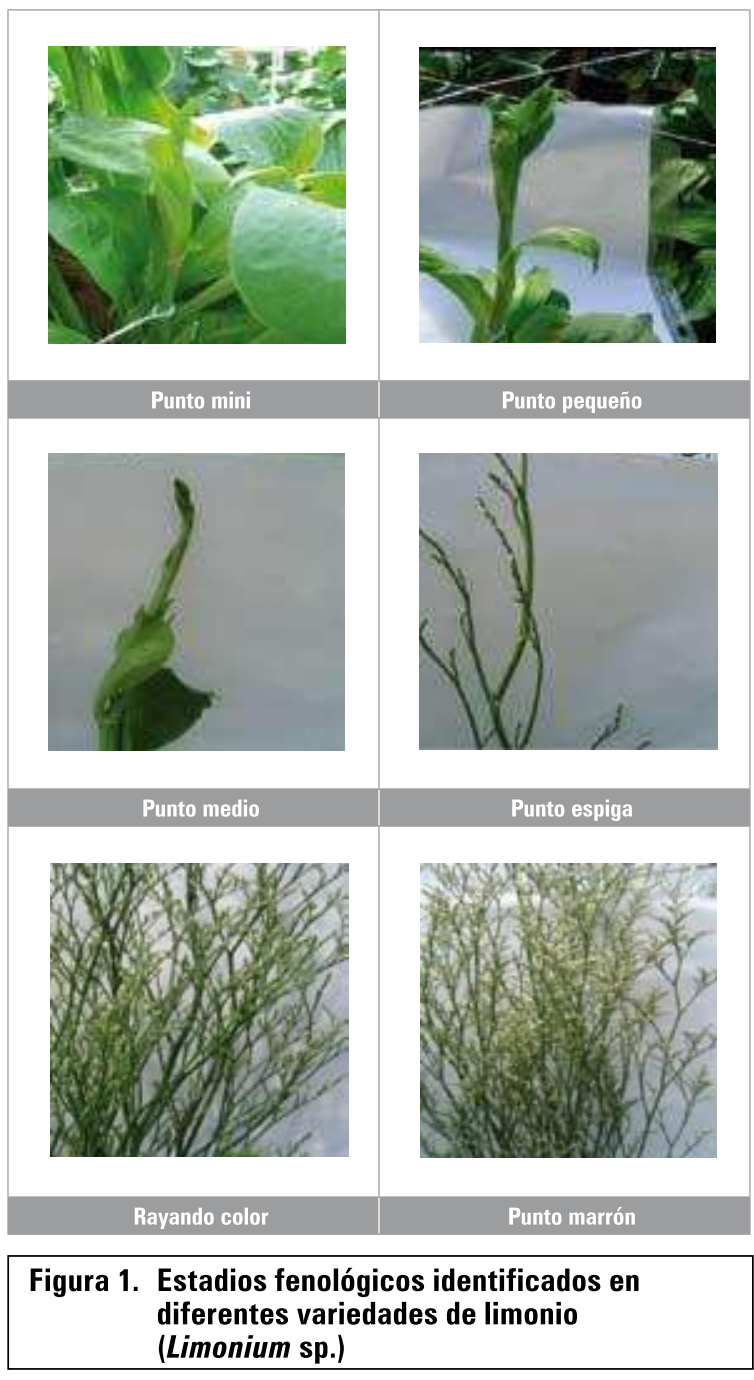

brote recién emergido y a su vez, el estado seis o punto marrón fue el más cercano al corte. Se marcó cada uno de ellos mediante la utilización de etiquetas de marcación, las cuales contenían información adicional como la ubicación de la planta y fecha de inicio de las mediciones. Para medir la longitud de los tallos o brotes se utilizó un flexómetro colocado de manera paralela a cada brote, colocando un extremo del mismo en la base de la planta, y el otro extremo hacia el ápice del brote, con lo cual se registró la altura de los tallos. Se monitoreó el tiempo necesario para alcanzar el punto de corte a partir de cada estadio fenológico, así como la duración de cada uno de los estados fenológicos de cada variedad. 
El experimento consistió en un ensayo bifactorial, con cinco variedades como primer factor y seis estadios fenológicos como factor adicional. Se utilizó un diseño completamente al azar y cinco replicaciones, tomando una planta como unidad experimental. La información, se analizó mediante un análisis de varianza (Anava) y la prueba de separación de promedios de Tukey $(P \leq 0,01)$. Adicionalmente se realizó un análisis de regresión para las curvas de crecimiento, con el programa SPSS versión 11.5.1.

\section{RESULTADOS Y DISCUSIÓN}

Acorde con el análisis de varianza, se presentaron diferencias altamente significativas $(P \leq 0,01)$ para los factores variedad y estado fenológico, así como para todas las combinaciones de estos factores. Entre las variedades estudiadas se encontró que 'Supreme white light' fue el material que presentó tallos florales más largos, seguido por 'Supreme blue night', 'Beltlaard', 'Safora dark blue', y por último 'Safora blue' (figura 2). Esta información es de interés para los cultivadores de flores, con miras a determinar el destino de la flor cosechada, dado que la elaboración de bouquets requiere de flores con diferentes longitudes, acorde con el tamaño de las flores acompañantes. Adicionalmente se requiere el contraste de las flores complementarias con las de limonio, por tanto se hace necesario considerar la longitud de sus tallos.

De acuerdo con el estado fenológico de partida para la toma de la información, en los dos primeros estadios, los brotes crecen de manera acelerada entre 36 y 43 d después de la aparición del estado fenológico, posteriormente, la velocidad del crecimiento disminuye de manera considerable (figura $3 \mathrm{~A}$ y $3 \mathrm{~B}$ ). Este modelo de crecimiento sigmoide o hiperbólico representa el incremento en altura en plantas cuyas ramas tienen un tipo de crecimiento definido (Taiz y Zeiger, 2006). Las ramas presentan un crecimiento lento, posteriormente el crecimiento en altura es logarítmico

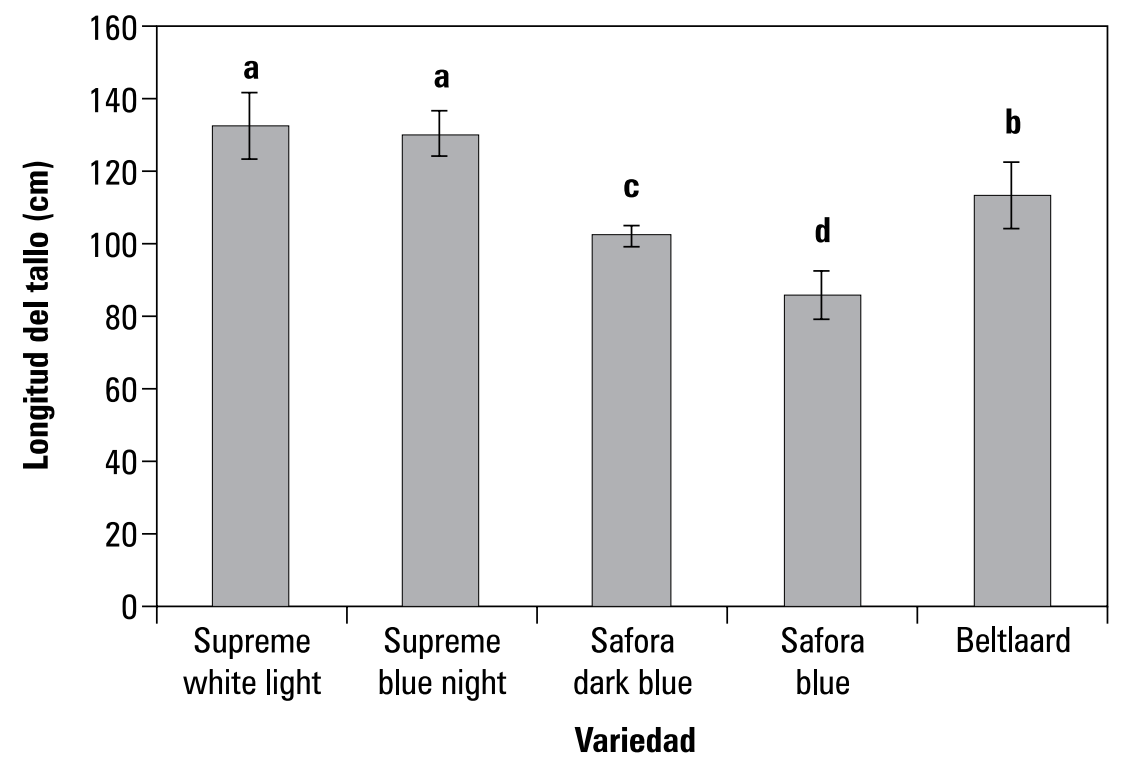

Figura 2. Longitud máxima del tallo en cinco variedades de limonio (Limonium sp.) cultivadas bajo invernadero. La barra sobre la columna indica la desviación estándar. Promedios con letras distintas indican diferencia significativa según la prueba de Tukey $(P \leq 0,01)$. 
y por último se presenta una fase estacionaria. Esta última fase corresponde a la aparición de las flores en los meristemos apicales, por tanto, las ramas no pueden crecer más debido a su crecimiento determinado. En la figura 3A se observa este modelo de crecimiento en los cinco materiales evaluados, sin embargo, la velocidad del crecimiento en cada una de ellas difiere en la medida en que el material es precoz como 'Safora blue' $y$ 'Beltlaard' o tardío como 'Safora dark blue', 'Supreme dark light' $y$ 'Supreme blue night'.

Tomando en consideración los estadios fenológicos avanzados (figuras 3B a 3F), en la medida en que se tomó la información a partir de estadios más avanzados, empieza a desaparecer de la figura la fase de crecimiento logarítmica, quedando sólo la fase estacionaria (figuras $3 \mathrm{E}$ a $3 \mathrm{~F}$ ). La aplicación práctica de esta información radica en el hecho que una vez identificados los diferentes estadios en las variedades evaluadas se debe esperar un comportamiento de las variedades acorde con la ruta que describen las figuras y así, es posible hacer la predicción del corte de la flor con base en la información consignada en la tabla 1, en la cual aparecen las ecuaciones de regresión para cada una de las curvas presentadas en la figura 3, así como el tiempo restante de corte para cada variedad, cuando se parte de cada uno de los estadios evaluados.

Las dos fechas importantes para la venta de flor cortada corresponden al día de las madres y el día de San Valentín. Mientras que en Europa el día de las madres se celebra el primer domingo de mayo, en Estados Unidos se celebra el segundo domingo del mismo mes (Escuela Internacional, 2007). En el día de San Valentín se celebra el día de los enamorados, el 14 de febrero. Por tanto, la proyección de los puntos de corte para dar cumplimiento a la exportación de la flor en estas dos fechas es motivo de una planificación minuciosa en las compañías colombianas productoras de flores para el mercado exterior.

El punto de corte es de gran relevancia para la calidad de bouquet, dado que en las plantas de limo- nio, las flores no continúan su apertura luego de cortadas. Adicionalmente se debe hacer énfasis en la importancia del color de la flor para lograr contraste con las flores accesorias del bouquet. Los colores de los materiales utilizados en este estudio correspondieron a blanco aterciopelado para 'Supreme white light', azul lavanda para 'Supreme blue night', púrpura profundo para 'Safora dark blue', púrpura-azul oscuro para 'Safora blue' y por último violeta para 'Beltlaard'.

Se debe resaltar que de los materiales evaluados, solo en 'Beltlaard' es posible el cambio de color de la flor a través de su tinción, para lo cual, la flor se corta con una semana de anticipación a la fecha de envío, con miras a tener el tiempo suficiente para teñir las flores. El procedimiento para tinte es reserva comercial de las empresas. Por otro lado, las flores que no se tinturan se cortan el mismo día de despacho. Al momento de corte, las flores de la variedad Beltlaard no han adquirido su color violeta característico, así que en ese punto presentan una tonalidad beige intenso, lo cual facilita el cambio artificial de color.

Con base en esta información y en la metodología empleada en el estudio, los cultivadores de las variedades evaluadas podrán realizar la planificación del momento de corte de la flor, en la medida que la flor pueda o no tinturarse. Además, la utilización de los estadios fenológicos como indicador morfológico para predecir el momento de corte representa una herramienta práctica en las labores de planificación. Se debe hacer hincapié en la determinación del estadio fenológico con base en la escala mostrada en la metodología, con el fin de partir de un estadio determinado y no de una fecha calendario, dado que el desarrollo de los brotes es altamente dependiente de factores del entorno, como la temperatura (Sitte et al., 2004). Es así, como temperaturas altas pueden promover una mayor tasa de crecimiento como consecuencia de un metabolismo acelerado y por tanto, pueden acortar el lapso de tiempo entre la aparición de un estadio fenológico y el momento de corte. 

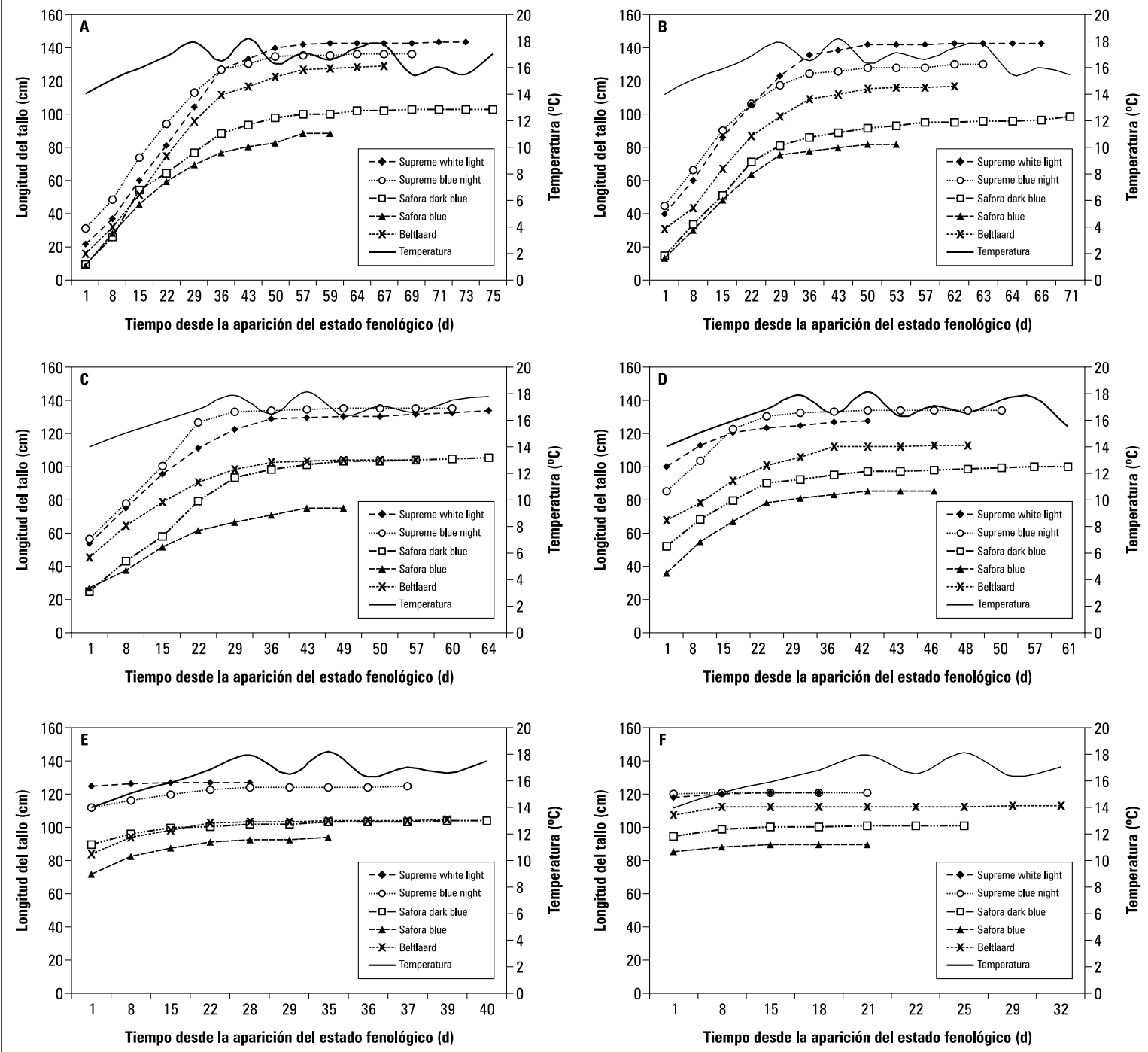

Figura 3. Curvas de crecimiento en cinco variedades de limonio (Limonium sp.) a partir de seis estadios fenológicos. A) A partir de punto mini; B) A partir de punto pequeño; C) A partir de punto medio D) A partir de punto espiga; E) A partir de rayando color; F) A partir de punto marrón.

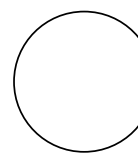

AGRADECIMIENTOS

Este estudio se desarrolló con el apoyo de la Dirección de Investigaciones (DIN) de la Universidad Pedagógica y Tecnológica de Colombia, en el marco del plan de trabajo del Grupo de Investigación Ecofisiología Vegetal, adscrito al programa de Ingeniería Agronómica de la Facultad de Ciencias Agropecuarias, Tunja. 


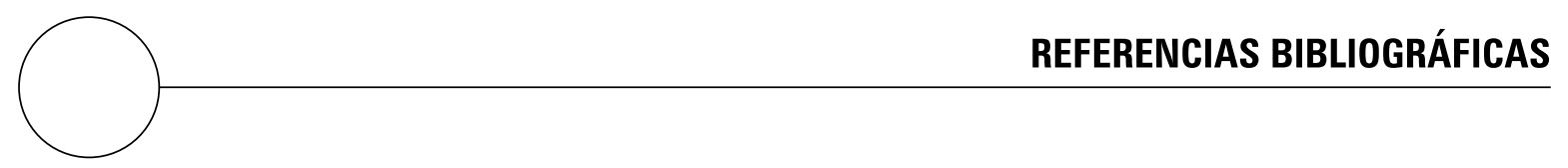

Asocolflores. 2007. Floricultura colombiana. Información general. En: http://www.asocolflores.org/asocolflores $/$ servlet $/$ Download? idExternalFile $=236 \&$ name =HOJA-DATOS-2008-7.pdf; consulta: octubre de 2008

Baltzer, J.L.; E.G. Reekie; H.L. Hewlin; P.D. Taylor y T.S. Boates. 2002. Impact of flower harvesting on the salt marsh plant Limonium carolinianum. Can. J. Bot. 80, 841-851.

Cárdenas, C.A.; I.F. Rivera; V.J. Flores R.; W. Piedrahita C. y B. Chaves C. 2006. Análisis de crecimiento en clavel estándar variedad 'Nelson' cultivado en sustratos. pp. 111-127. En: Florez R., V.J.; A.D.L.C. Fernández; D. Miranda L.; B. Chaves C. y J.M. Guzmán P. (eds.). Avances sobre fertirriego en la floricultura colombiana. Facultad de Agronomía, Universidad Nacional de Colombia; Unibiblos, Bogotá.

Casierra-Posada, F. y D.L. Moreno. 2007. Efecto del estrés por sombra sobre la producción en plantas de limonio (Limonium sp. cv. Bluestream). Rev. Colomb. Cienc. Hortic. 1(2), 236-245.

Chamorro, A.H.; S.L. Martínez; J.C. Fernández; T. Mosquera. 2007. Evaluación de diferentes concentraciones de algunos reguladores de crecimiento en la multiplicación y enraizamiento in vitro de Limonium var. Misty blue. Agron. Colomb. 25(1), 47-53.

Chaparro, L.A.; A. Farías A.; B. Chaves C.; D. Miranda L. y V.J. Flores R. 2006. Análisis de crecimiento en rosa variedad 'Charlotte' en los sistemas de cultivo en sustrato y en suelo. pp. 77-90. En: Florez R., V.J.; A.D.L.C. Fernández; D. Miranda L.; B. Chaves C. y J.M. Guzmán P. (eds.). Avances sobre fertirriego en la floricultura colombiana. Facultad de Agronomía, Universidad Nacional de Colombia; Unibiblos, Bogotá.

Doi, M. y M.S. Reid. 1995. Sucrose improves the postharvest life of cut flowers of a hybrid Limonium. HortScience 30(5), 1058-1060.

Dufour, L. y V. Guérin. 2002. Growth, developmental features and flower production of Anthurium andreanum Lind. in tropical conditions. Scientia Hort. 98, 25-35.
Escher, F. 1996. Kulturbeschreibungen. pp. 414-420. En: Escher, F.; H.-C. Gudehus; L. Mayr; W. Mezinger; P. Rehrmann y H. Thomale (eds.). Schnittblumenkulturen. Ulmer Verlag, Stuttgart, Alemania.

Escuela Internacional. 2007. Día de la madre: Primer domingo de mayo. En: http://www.escuelai.com/ spanish_culture/fiestas_espanolas/diadelamadre. html; consulta: octubre de 2008.

Farías, A.; L.A. Chaparro; A. Campos; B. Chaves y V.J. Flores. 2006. Curvas de crecimiento de rosa en sistema de cultivo sin suelo en la Sabana de Bogotá. pp. 91-110. En: Florez R., V.J.; A.D.L.C. Fernández; D. Miranda L.; B. Chaves C. y J.M. Guzmán P. (eds.). Avances sobre fertirriego en la floricultura colombiana. Facultad de Agronomía, Universidad Nacional de Colombia; Unibiblos, Bogotá.

Farina, E.; C. Dalla Guda y E. Scordo. 2000. Effects of low temperatures and photoperiod on flowering of Limonium gmelinii. Acta Hort. 541, 193-199.

Fuentes, V. 1992a. Influencia de los factores climáticos y la fase fenológica en el contenido de metabolitos secundarios en las plantas. Experiencias en Cuba. p. 7. En: Resúmenes del seminario-taller provincial "La agrometeorología al servicio de la agricultura". La Habana.

Fuentes, V. 1992b. Los estudios fenológicos en plantas medicinales en cuba. Experiencias en Cuba. p. 6. En: Resúmenes del seminario-taller provincial "La agrometeorología al servicio de la agricultura". La Habana.

Ghersa, C.M. y J.S. Holt. 1995. Using phenology prediction in weed management: a review. Weed Res. 35, 461-470.

Shillo, R. 1976. Control of flower initiation and development of statice (Limonium sinuatum) by temperature and daylength. Acta Hort. 64, 197-203.

Sitte, P.; E.W. Weiler; J.W. Kadereit; A. Bresinsky y C. Körner. 2004. Strassburger: Tratado de botánica. 35a ed. Ediciones Omega, Barcelona, España.

Taiz, L. y E. Zeiger. 2006. Plant physiology. Sinauer Associates, Inc., Publishers, Sunderland, MA. 